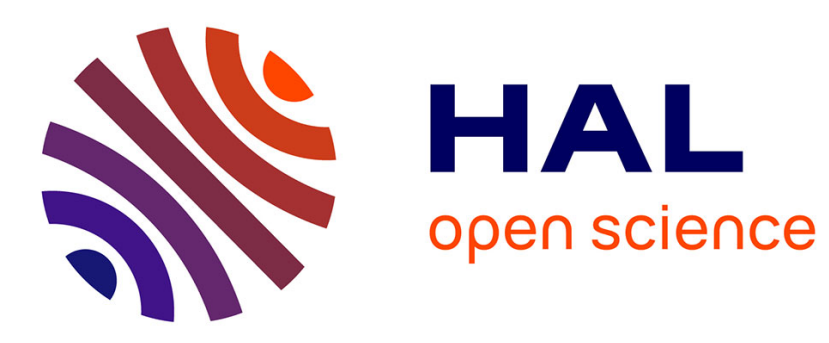

\title{
Numerical analysis of the drag on a rigid body in an immersed granular flow
}

Nathan Coppin, Matthieu Constant, Jonathan Lambrechts, Frédéric Dubois, Vincent Legat

\section{- To cite this version:}

Nathan Coppin, Matthieu Constant, Jonathan Lambrechts, Frédéric Dubois, Vincent Legat. Numerical analysis of the drag on a rigid body in an immersed granular flow. Computational Particle Mechanics, 2021, 10.1007/s40571-021-00418-w . hal-03269423

\section{HAL Id: hal-03269423 https://hal.science/hal-03269423}

Submitted on 28 Jun 2021

HAL is a multi-disciplinary open access archive for the deposit and dissemination of scientific research documents, whether they are published or not. The documents may come from teaching and research institutions in France or abroad, or from public or private research centers.
L'archive ouverte pluridisciplinaire HAL, est destinée au dépôt et à la diffusion de documents scientifiques de niveau recherche, publiés ou non, émanant des établissements d'enseignement et de recherche français ou étrangers, des laboratoires publics ou privés. 


\title{
Numerical analysis of the drag on a rigid body in an immersed granular flow
}

\author{
Nathan Coppin ${ }^{1}\left(\mathbb{D} \cdot\right.$ Matthieu Constant ${ }^{1} \cdot$ Jonathan Lambrechts $^{1} \cdot$ Frédéric Dubois $^{2,3} \cdot$ Vincent Legat $^{1}$
}

\begin{abstract}
The drag exerted on an object moving in a granular medium is subject to many studies: in dry grains, it depends on the stress - often due to gravity - inside the grains. For Froude numbers less than 10, the drag is independent of the velocity. In immersed grains, it is proportional to the apparent weight of the grains and it depends on both velocity and fluid viscosity. In this paper, the drag on a cylinder in dry and immersed granular flow is simulated with a multi-scale FEM-DEM model. The Janssen stress saturation effect before the flow and velocity independence of the drag are both reproduced. The semi-circular orientation of the stress field supports the hypothesis of a spherical zone of influence of the cylinder. This orientation does not significantly change upon flowing. The results show the velocity independence of the drag is due to particle friction. In the immersed case, the fluid contribution is negligible on its own. A dimensional analysis suggests that shear thickening should be taken into account. The transition between the quasi-static and the fluidized regime is accompanied by a decrease of the stress field downstream of the cylinder and a change in the shape of the shear zone.
\end{abstract}

Keywords Granular drag · Janssen effect · Nonsmooth contact dynamics · Unresolved model · MigFlow

\section{Introduction}

Granular flows exhibit complex behaviours, and are the subject of many experimental, theoretical and numerical studies. A quantity of interest is the drag force exerted on an object that is intruding such a material. This quantity is involved in many practical and industrial problems such as the design of mixing and plowing devices [51], or anti-avalanche pro-

Nathan Coppin

nathan.coppin@uclouvain.be

Matthieu Constant

matthieu.constant@uclouvain.be

Jonathan Lambrechts

jonathan.lambrechts@uclouvain.be

Frédéric Dubois

frederic.dubois@umontpellier.fr

Vincent Legat

vincent.legat@uclouvain.be

1 Institute of Mechanics, Materials and Civil Engineering, Applied Mechanics and Mathematics, Avenue Georges Lemaitre 4-6 bte L4.05.02, Louvain-la-Neuve, Belgium

2 LMGC, Univ. Montpellier, CNRS, Montpellier, France

3 MIST, Univ. Montpellier, CNRS, IRSN, Montpellier, France tections [44]. Oftentimes, a surrounding fluid significantly modifies the dynamic or static behaviour of the material.

In the dry case, experiments on the granular drag begun in the early 1970's with Wieghardt's work. He noticed that the drag did not mainly depend on the flow velocity, but rather on the shape and size of the intruder [51]. Further experiments established that at moderate velocities, the velocity had no impact on the drag force. The latter mostly depends on the object shape and size and on gravitational stress inside the material $[5,16,24,53]$. This is because the motion of the intruder implies a reorganisation of the spatial structure of the grains, which in turn depends on geometrical parameters and on the gravitational stress that maintain the grains together. Hence, the flow can be characterised with the Froude number $F r=V / \sqrt{g L}$ with $V$ the flow velocity, $g$ the acceleration due to gravity and $L$ a characteristic length - e.g. the intruder diameter, the hydraulic radius of the channel or the intruder's penetration depth in the granular bed, depending on the geometry of the problem. At Froude number greater than 10, the granular material becomes fluidized. Then, the transfer of momentum between the grains and the object dominates yielding a square velocity dependence $[24,45,49]$.

Several experiments were led in the presence of an interstitial fluid. When the material is not saturated, the velocity 
dependence on the drag force is similar to the dry case, although mechanisms such as cohesion and contact lubrication have an influence [9]. On the other hand, when the grains are fully immersed, the buoyancy due to the fluid decreases the apparent weight of the grains. This decreases the value of the drag force [19] and the Froude number, leading to an earlier fluidization. The ratio between the drag force and the average weight of the grains on the object was observed to vary as a power law of a so-called viscous number $J=\eta \dot{\gamma} / P$ with $\eta$ the viscosity of the fluid, $\dot{\gamma}$ the shear rate and $P$ the pressure in the granular material $[6,37]$.

Theoretical models for granular flows exist for different regimes such as granular gases [14,33] or quasi-static flows [13,39]. However, establishing a unified approach independent of the flow regime remains difficult [40], although there are progresses concerning the drag [24]. This struggle, in addition to the difficulty for experimental studies to have access to grain scale phenomena, raises the interest for numerical methods. These techniques allow better insight on the physics of the grains - such as the velocity field, the contact network or the stress tensor - and control over the physical properties - such as friction - although they require some calibration.

Several methods exist to simulate divided media. Smooth methods, launched in the 1970's by Cundall and Strack, are explicit time-stepping methods that rely on forcedisplacement laws for the resolution of the contacts [20]. On the other hand, nonsmooth methods consider instantaneous and unilateral contacts. This implies a discontinuity in the velocities of the particles and prevents the particles from overlapping. The event-driven method (ED) assumes binary contacts and solves them sequentially, jumping in time from one contact to another. It is suitable for granular gases $[3,12]$. The Nonsmooth Contact Dynamics (NSCD) deals with the discontinuity of velocities with an implicit formulation of the contact laws, which amounts to a balance of momentum, coupled to a Coulomb law for dry friction. This event-capturing scheme has a constant time step, over which all contacts are solved at once. It is done by using an iterative procedure like Non Linear Gauss Seidel iterations [30]. This implicit timestepping resolution has several advantages: it avoids inelastic collapse when the time between collisions vanishes, it allows to use a larger time step than explicit methods, and it eliminates the need for global damping in smooth methods to reach an equilibrium state. Although static and dynamic situations are accurately rendered, the method still faces problems, such as hyperstaticity or the impossibility to reach exactitude both in position and velocity [1].

As far as fluid-grain mixtures are concerned, different approaches exist. The simplest models make a continuum assumption both for the fluid and the granular phases [25]. They result in a low computational cost, but ignore discrete interactions between the grains themselves and between the grains and the boundaries. Direct simulations combine the Lagrangian methods cited above for the grains with a method for the fluid which explicitly computes the flow around each grain, $[27,38,52]$. Although very accurate, those methods have a very high cost, because the element size is smaller than the one of the grains. Multi-scale methods combine the resolution of the fluid at a coarse scale, solving volumeaveraged Navier-Stokes equations, with DEM methods for the resolution of the solid phase at the grain scale $[22,48]$. The MigFlow ${ }^{1}$ software makes use of such an hybrid approach.

The goal of this paper is to simulate the drag force exerted on a cylinder by flowing granular medium, dry or immersed, across a range of various properties such as fluid density and dynamic viscosity or particle friction coefficients. To do so, the frictional contact solver for the granular phase as well as a channel simulation geometry are first validated against a physical experiment on the drag exerted on a cylinder in a dry granular flow. Then, the impact of the friction coefficients between the particles and the particles and the walls is investigated. Finally, the presence of an interstitial fluid is simulated for different values of density and viscosities.

\section{Numerical method}

The MigFlow software solves the granular phase with the NSCD method, and the volume averaged Navier-Stokes equations for the fluid phase with a Finite Element Method. Both solvers are coupled using a predictor-corrector scheme. This section first describes the NSCD and its implementation. Then, the fluid problem and its Finite Element Method resolution are explained, followed by the description of the predictor-corrector scheme.

\subsection{Granular phase solver}

Nonsmooth contact dynamics have been applied to a wide variety of problems, be they discrete (granular materials or stone masonry) or continuous (deep-drawing) in nature.

In this way, its adaptability to different body shapes, time integration schemes and numerical techniques has proven useful. This paper only aims at presenting the simpler version of the method for spheres used in MigFlow, but more complete descriptions of the various features can be found in $[1,30,31]$.

\subsubsection{Unilateral contact}

Consider a pair of spherical bodies in an upcoming contact, such as in Fig. 1. The body $j$ is called the candidate, and $i$ the antagonist. Since these bodies are convex, there exists a

\footnotetext{
$\overline{1 \text { www.migflow.be. }}$
} 


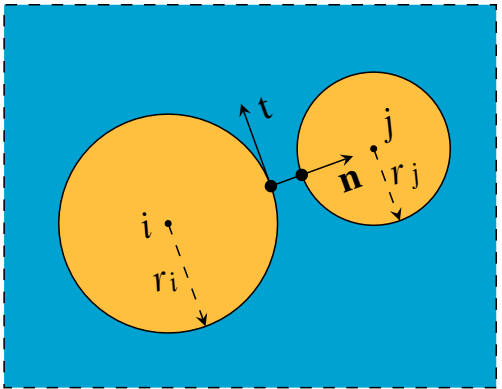

Fig. 1 The candidate body $j$ and the antagonist $i$ in potential contact

pair of contact points on their boundaries as well as a unique normal vector from the candidate to the antagonist in the centre-to-centre direction. A local frame can be constructed by associating to the normal vector $\mathbf{n}$ a set of orthonormal vectors, as described in Fig. 1. The focus is put on the relative velocity of the contact points $\mathbf{U}$. Its normal component is $U_{N}$ and its tangential ones are $\mathbf{U}_{T}$. The contact is assumed to be unilateral, implying that $i$ and $j$ do not interpenetrate. Since this results in velocity discontinuities, it is necessary to assume that the relative velocity is a locally bounded variation function. It implies that at each time $t$ the function has a left limit $\mathbf{U}\left(t^{-}\right)=\mathbf{U}^{-}$and a right limit $\mathbf{U}\left(t^{+}\right)=\mathbf{U}^{+}$[35]. The non-interpenetrability is expressed by stating that the distance between the contact points $\mathrm{d}=\left\|\mathbf{x}_{j}-\mathbf{x}_{i}\right\|-\left(r_{i}+r_{j}\right)$ should remain positive. It is ensured by specifying that the normal component of the reaction impulse $P_{N}$ is positive and vanishes as soon as the contact is broken. This leads to the so-called Signorini condition:

$\mathrm{d} \geq 0, \quad P_{N} \geq 0, \quad \mathrm{~d} P_{N}=0$.

From the following relationship:

$\frac{d \mathrm{~d}}{d t}=U_{N}$

it is possible to write the Signorini condition in terms of velocity:

$U_{N}^{+} \geq 0, \quad P_{N} \geq 0, \quad U_{N}^{+} P_{N}=0$.

The Signorini condition is a multi-application and $U_{N}^{+}$cannot be explicitly deduced from $P_{N}$. A shock law is necessary to close the system. A newton law with a null restitution coefficient is chosen to cancel the relative velocity after the contact:

$U_{N}^{+}=0$.

This implies perfectly inelastic collisions between the particles, which proves useful to dissipate energy and avoid persistent vibrations without the use of global damping. As far as friction is concerned, it is modeled with a Coulomb law for dry friction. The frictional impulse is bounded by a threshold depending on the normal reaction impulse. When sliding occurs, it is opposite to the sliding velocity and has a magnitude equivalent to the normal impulse times the friction coefficient:

$$
\left\|\mathbf{P}_{T}\right\| \leq \mu P_{N}, \quad\left\|\mathbf{U}_{T}^{+}\right\| \neq 0 \Rightarrow \mathbf{P}_{T}=-\mu P_{N} \frac{\mathbf{U}_{T}^{+}}{\left\|\mathbf{U}_{T}^{+}\right\|},
$$

with $\mu$ the coefficient of friction.

\subsubsection{Equations of motion}

The sets of coordinates and velocities of the grains in the laboratory frame of reference are denoted as:

$\mathbf{q}=\left(\begin{array}{c}\vdots \\ \mathbf{x}_{i} \\ \boldsymbol{\theta}_{i} \\ \mathbf{x}_{j} \\ \boldsymbol{\theta}_{j} \\ \vdots\end{array}\right) \quad \frac{d \mathbf{q}}{d t}=\mathbf{u}=\left(\begin{array}{c}\vdots \\ \mathbf{u}_{i} \\ \omega_{i} \\ \mathbf{u}_{j} \\ \omega_{j} \\ \vdots\end{array}\right)$,

where $\mathbf{x}_{k}, \boldsymbol{\theta}_{k}$ are the position and Euler angles vectors of particle $k$ and $\mathbf{u}_{k}, \boldsymbol{\omega}_{k}$ its velocity and angular velocity vectors. The equations of motion for the particles are obtained from Newton's second law:

$$
\begin{aligned}
\mathbf{M} \frac{d \mathbf{u}}{d t} & =\mathbf{f}_{e}+\mathbf{r} \\
\mathbf{q}(t) & =\mathbf{q}(\tau)+\int_{\tau}^{t} \mathbf{u} d t .
\end{aligned}
$$

with $\mathbf{M}$ the mass matrix, $\mathbf{f}_{e}$ the explicitly known external forces such as gravity or fluid-grain interaction forces, and $\mathbf{r}$ the sum of all reaction forces due to the contacts between the particles. The problem consists in finding all contact reactions for all the contacts during a given time step. To this end, it is necessary to iteratively solve each contact by switching to its local frame of reference and applying the contact laws before switching back to the global frame of reference. The switching to the local contact frame of reference is performed using a transformation $\mathbf{H}^{*}(\mathbf{x})$ :

$\mathbf{U}=\mathbf{H}^{*}(\mathbf{x}) \mathbf{u}$,

where $\mathbf{x}$ represents the set of the positions $\mathbf{x}_{k}$ of the particles. From an equality of power expressed in the two frames, it can be shown that the reciprocal transformation is given by its transpose $\mathbf{H}(\mathbf{x})$ [1]. 


\subsubsection{Time discretisation}

In a dense granular material, the time between two successive contacts vanishes. Since the time at which each contact occurs is ill-defined, a time stepping method such as NSCD aims to solve all the contacts occurring in a given time interval $\Delta t$ at once. This consist in finding the values of $\mathbf{q}$ and $\mathbf{u}$ at the end of the time step, given their value at the beginning. Various approximation formulas can be established to estimate an intermediary state of these variables to be used in the computation, see [30], but as it is not done here, it shall not be discussed.

Integrating the equations of motion with an Euler explicit scheme for the external forces, and an implicit one for the reaction forces and the velocity in the second expression leads to:

$$
\begin{aligned}
\mathbf{M}\left(\mathbf{u}^{n+1}-\mathbf{u}^{n}\right) & =\mathbf{f}_{e}^{n} \Delta t+\mathbf{p}^{n+1} \\
\mathbf{q}^{n+1} & =\mathbf{q}^{n}+\mathbf{u}^{n+1} \Delta t,
\end{aligned}
$$

$\mathbf{p}^{n+1}$ being the sum of all the impulses over the time-step due to the contacts.

The matrices $\mathbf{H}^{*}(\mathbf{x})$ that transform the variables are considered constant over the time step because the change in $\mathbf{x}$ over the time step is small with respect to the curvature of the bodies [30].

\subsubsection{Resolution of a contact}

The contact laws detailed earlier are expressed in the local frame. Consequently, if a contact is to be solved, it is first necessary to switch to this frame. Once the switch is performed, the following set of uncoupled equations is to be solved:

$$
\begin{aligned}
& \left\{\begin{array}{l}
U_{N}^{n+1}=U_{f l N}+W_{N N} P_{N}^{n+1} \\
W_{N N}=m_{j}^{-1}+m_{i}^{-1}
\end{array}\right. \\
& \left\{\begin{array}{l}
\mathbf{U}_{T}^{n+1}=\mathbf{U}_{f l T}+\mathbf{W}_{T T} \mathbf{P}_{T}^{n+1} \\
\mathbf{W}_{T T}=\operatorname{diag}\left(W_{N N}+r_{i}^{2} I_{i}^{-1}+r_{j}^{2} I_{j}^{-1}\right)
\end{array}\right.
\end{aligned}
$$

with $m_{k}$ and $I_{k}$ the mass and inertia of particle $k$, and $W_{N N}$ and $\mathbf{W}_{T T}$ the components of a matrix $\mathbf{W}$ associated to the normal and tangential directions, respectively. This matrix W has dimensions $\mathrm{kg}^{-1}$ and results from the inverse of the mass matrix multiplied to the left and to the right by the switching matrices $\mathbf{H}^{*}$ and $\mathbf{H}$, respectively. The free local velocity $\mathbf{U}_{f l}$ represents the relative velocity of the particles due to the external forces and to the other contacts implying the two particles. The perfectly inelastic shock law and the

\section{Algorithm 1: Contact resolution}

- Initiate the contact:

$$
\begin{aligned}
\mathbf{n} & =\frac{\mathbf{x}_{j}-\mathbf{x}_{i}}{\left\|\mathbf{x}_{j}-\mathbf{x}_{i}\right\|}, \perp \mathbf{t} \in \mathbb{R}^{2 \times 3} \\
c_{i} & =\frac{m_{j}}{m_{i}+m_{j}}, \quad c_{j}=\frac{m_{i}}{m_{i}+m_{j}} \\
a & =\frac{I_{i}}{m_{i} r_{i}^{2}}=\frac{I_{j}}{m_{j} r_{j}^{2}}=\frac{2}{5}
\end{aligned}
$$

Construct

the local frame Compute redistribution coefficients Compute the moment of inertia factor

- Compute the relative velocities:

$$
\begin{aligned}
U_{N} & \leftarrow \delta U_{N}^{\text {old }}+\mathbf{n} \cdot\left(\mathbf{u}_{j}-\mathbf{u}_{i}\right) \\
\mathbf{U}_{T} & \leftarrow \delta \mathbf{U}_{T}^{\text {old }}+\mathbf{t} \cdot\left(\mathbf{u}_{j}-\mathbf{u}_{i}\right) \\
& +\mathbf{t} \cdot\left[\left(\boldsymbol{\omega}_{i} r_{i}+\omega_{j} r_{j}\right) \times \mathbf{n}\right]
\end{aligned}
$$

Remove previous corrections

- Solve the normal component:

$$
\delta U_{N} \leftarrow \max \left(0, U_{N}-\frac{\mathrm{d}}{\Delta t}\right)
$$

- Solve the tangential components:

$$
\delta \mathbf{U}_{T} \leftarrow \min \left(\left\|\mathbf{U}_{T}\right\|, \frac{a+1}{a} \mu \delta U_{N}\right) \frac{\mathbf{U}_{T}}{\left\|\mathbf{U}_{T}\right\|}
$$

- Correct the velocities:

$$
\begin{array}{ll}
\delta U_{N} \leftarrow \delta U_{N}-\delta U_{N}^{\text {old }} & \begin{array}{l}
\text { Include } \\
\text { previous } \\
\text { corrections }
\end{array} \\
\delta \mathbf{U}_{T} \leftarrow \delta \mathbf{U}_{T}-\delta \mathbf{U}_{T}^{\text {old }} & \\
\mathbf{u}_{i} \leftarrow \mathbf{u}_{i}-c_{i}\left[\delta U_{N} \mathbf{n}+\frac{a}{a+1} \mathbf{t}^{*} \cdot \delta \mathbf{U}_{T}\right] & \begin{array}{l}
\text { Transform } \\
\text { local } \\
\text { corrections } \\
\text { into } \\
\text { global ones }
\end{array} \\
\mathbf{u}_{j} \leftarrow \mathbf{u}_{j}+c_{j}\left[\delta U_{N} \mathbf{n}+\mathbf{t}^{*} \cdot \frac{a}{a+1} \delta \mathbf{U}_{T}\right] & \\
\boldsymbol{\omega}_{i} \leftarrow \boldsymbol{\omega}_{i}+\frac{c_{i}}{r_{i}} \frac{1}{a+1} \mathbf{t}^{*} \cdot\left(\begin{array}{rr}
0 & 1 \\
-1 & 0
\end{array}\right) \cdot \delta \mathbf{U}_{T} & \\
\boldsymbol{\omega}_{j} \leftarrow \boldsymbol{\omega}_{j}+\frac{c_{j}}{r_{j}} \frac{1}{a+1} \mathbf{t}^{*} \cdot\left(\begin{array}{rr}
0 & 1 \\
-1 & 0
\end{array}\right) \cdot \delta \mathbf{U}_{T}
\end{array}
$$

coulomb friction yield:

$$
\begin{aligned}
& P_{N}^{n+1}=-U_{f l N} / W_{N N} \\
& \mathbf{P}_{T}^{n+1}=\min \left(\mu P_{N}^{n+1},\left\|\mathbf{P}_{\text {stick }}\right\|\right) \frac{\mathbf{P}_{\text {stick }}}{\left\|\mathbf{P}_{\text {stick }}\right\|},
\end{aligned}
$$


with $\mathbf{P}_{\text {stick }}=-\mathbf{W}_{T T}^{-1} \mathbf{U}_{f l T}$ the impulse that cancels the tangential relative velocity.

Each contact is solved based on the above procedure. In practice, the impulses are directly computed as relative velocity corrections, and then the velocities of the grains are modified accordingly, as pictured in Algorithm 1. Contacts with the boundaries are solved in the same way, except that the boundaries are treated as having infinite mass. The moment of inertia factor has a value of 0.4 for solid spheres, but it can be tuned to match a given degree of hollowness of the grains. The previous corrections are canceled and then retaken into account for convergence purposes.

\subsubsection{Collection of grains}

When solving the contacts for a collection of grains, several difficulties arise. First, the velocity corrections due to the yet unsolved contacts are unknown. Furthermore, the resolution of one contact may affect a previously solved contact. To overcome these issues, an iterative procedure based on a queue is used. First, all possible contacts are detected with the help of an octree, and are inserted into the queue. Then, each contact of the queue is solved in turn. When a contact is solved, it is removed from the queue, and the contacts that imply one of the particles of the solved contact are put back in, if they are not already. If the difference between the previous corrections and the current ones times the time step are inferior to a given geometrical tolerance:

$\delta U_{N} \Delta t<\zeta$ and $\left\|\delta \mathbf{U}_{T}\right\| \Delta t<\zeta$,

then the contacts that imply the particles are not reinserted. Convergence is reached when the queue is empty. To accelerate this process, velocity corrections from the last time step are used as a first guess for the current one. Since in static situations, contacts are persistent and grain velocities do not change much, this results in a significant speed-up with equivalent accuracy [4]. Even then, convergence is not guaranteed for different reasons. One of them is that the kinematic constraints due to the constant approximation of the local frame of reference can be too large [31]. Since nonconvergence results in accuracy loss, a recursive adaptative strategy on the time step was implemented to deal with the above reason. When convergence cannot be reached, the current time-step is split in two sub time-steps that are solved with half the tolerance. To avoid infinite splitting when the non-convergence is due to another reason, a maximum number of splits is specified. When convergence is reached for all sub time-steps, the computation can go on to the next time-step.

\subsection{Fluid phase solver}

To simulate an interstitial fluid, the multi-scale approach allows to smooth the discontinuities due to the grains from the fluid point of view. The precise flow at the surface of the grains, on the other hand, is neglected. To do this, the fluid fields are replaced by their average on a given control volume, weighted by the fluid volume fraction over that control volume: the porosity [8].

The Navier-Stokes equations for an incompressible flow become:

$$
\begin{aligned}
& \frac{\partial \phi}{\partial t}+\nabla \cdot \mathbf{v}=0 \\
& \frac{\partial \rho_{f} \mathbf{v}}{\partial t}+\nabla \cdot \frac{\rho_{f} \mathbf{v} \mathbf{v}}{\phi}=\nabla \cdot\left(2 \eta \phi \mathbf{d}\left(\frac{\mathbf{v}}{\phi}\right)-p \mathbf{I}\right)+\mathbf{f}+\phi \rho_{f} \mathbf{g},
\end{aligned}
$$

where $\mathbf{v}$ is the volume-averaged fluid velocity, $\rho_{f}$ its density, $\eta$ its dynamic viscosity, $\mathbf{d}$ the deformation rate tensor, $\mathrm{p}$ the pressure, $\mathbf{I}$ the identity tensor, $\mathbf{f}$ the fluid-grain interaction force, and $\mathbf{g}$ the acceleration due to gravity. These equations are solved with a PSPG-SUPG stabilised P1-P1 Finite Element Method that is corrected for incompressibility with a LSIC term [17]. The currently used volume-averaging process requires the elements to be larger than the grains, but progress has been made that may suppress this constrain [15]. However, in this work the focus is put on the granular phase and an accuracy larger than the grain scale is sufficient for the fluid phase. Therefore, linear interpolation functions are satisfactory, and give good computational performances because of their simplicity. As for the fluid-grain interaction force $\mathbf{f}$, it only accounts for the pressure gradient and the drag contribution, neglecting other ones, such as the lift:

$\mathbf{f}=-\left.V_{p} \nabla p\right|_{\mathbf{x}}-\gamma\left(\mathbf{u}-\left.\frac{\mathbf{v}}{\phi}\right|_{\mathbf{x}}\right)$,

where $V_{p}$ is a vector containing the volumes of the grains, and where the drag component $\gamma$ coefficient follows an empirical formula that has been adapted to take into account the porosity $[41,50]$ and the Reynolds number so that it can be valid at different flow regimes [21]:

$\gamma=\left.\phi^{-1.8}\right|_{\mathbf{q}} \frac{\pi d^{2}}{4} \frac{\rho_{f}}{2}\left(0.63|| \mathbf{u}-\left.\frac{\mathbf{v}}{\phi}\right|_{\mathbf{x}}||^{\frac{1}{2}}+\left[\frac{\eta}{\left.d \rho_{f} \phi\right|_{\mathbf{x}}}\right]^{\frac{1}{2}}\right)$

This interaction force was validated in the MigFlow software for Stokes clouds of glass beads falling in a viscous fluid [18].

The resolution of the fluid phase rests on the following implicit time scheme:

$$
\frac{\phi^{n}-\phi^{n-1}}{\Delta t}+\nabla \cdot \mathbf{v}^{n+1}=0
$$




$$
\begin{aligned}
\rho_{f}\left(\frac{\mathbf{v}^{n+1}-\mathbf{v}^{n}}{\Delta t}+\nabla \cdot \frac{\mathbf{v}^{n+1} \mathbf{v}^{n}}{\phi^{n}}\right)= & \nabla \cdot 2 \eta \phi^{n} \mathbf{d}^{n+1}-\phi^{n} \nabla p^{n+1} \\
& +\left(\mathbf{f}^{n+1}+\left.V_{p} \nabla p^{n+1}\right|_{\mathbf{x}^{n+1}}\right) \\
& +\phi^{n} \rho_{f} \mathbf{g}
\end{aligned}
$$

\subsubsection{Fluid-grain coupling scheme}

For the sake of computational convenience, the fluid and the granular solvers are explicitly coupled. A predictor corrector scheme, detailed in [17], is used to improve the overall stability. However, with such an explicit coupling, the interaction force is still unstable. For this reason, the latter is computed with a Patankar scheme. Instead of using $\mathbf{u}^{n}$ to compute the interaction force, a prediction is made based on external forces at time $n+1$ and contact forces at time $n$ :

$$
\begin{aligned}
\frac{\mathbf{u}^{*}-\mathbf{u}^{n}}{\Delta t} & =\mathbf{g}+\mathbf{M}^{-1}\left(\mathbf{f}^{n+1}+\frac{\mathbf{p}^{n}}{\Delta t}\right) \\
\mathbf{f}^{n+1} & =-\left.V_{p} \nabla p^{n+1}\right|_{\mathbf{x}^{n}}-\gamma^{n}\left(\mathbf{u}^{*}-\left.\frac{\mathbf{v}^{n+1}}{\phi^{n}}\right|_{\mathbf{x}^{n}}\right)
\end{aligned}
$$

\section{Validation}

To validate the grain solver, the numerical model is confronted to a physical experiment performed by Chehata et al. [16]. They measured the drag force exerted on a cylinder inside a gravity-driven granular flow, and observed that it did not depend on the flow velocity.

The experimental apparatus, depicted in Fig. 2 left, consists of a vertical hopper through which a cylinder of diameter $D=38.1 \mathrm{~mm}$ is placed at a position $\mathbf{x}_{c}$. The top of the hopper is connected to a particle tank, while an aperture of variable size at its bottom controls the flow. The system is filled with glass particles with radius $r=3 \mathrm{~mm}$ and density $\rho_{p}=2500 \mathrm{~kg} / \mathrm{m}^{3}$. In the simulations, a small polydispersity based on a uniform distribution in the range $0.95 r-1.05 r$ was introduced to prevent crystallisation effects. The coefficients of friction are $\mu_{p}$ between the particles and $\mu_{w}$ between the particles and the walls. For the sake of simplicity, the coefficient of friction between the particles and the cylinder is also $\mu_{w}$. To be convenient with the FEM used to solve the presence of a fluid in further simulations, the cylinder is discretised as an octogonal prism. Instead of a large reservoir, particles are regularly reloaded when the level of the grains goes below a given limit, reducing computational costs. The number of injected particles is sufficiently small to make their impact on the force measured on the cylinder negligible. Finally, a channel geometry depicted in Fig. 2 right is tested. In this case, the particles rest on a fixed bed of particles (represented in red in Fig. 2 right), in a similar way to what is done in [32]. In addition to be less CPU time consuming, this

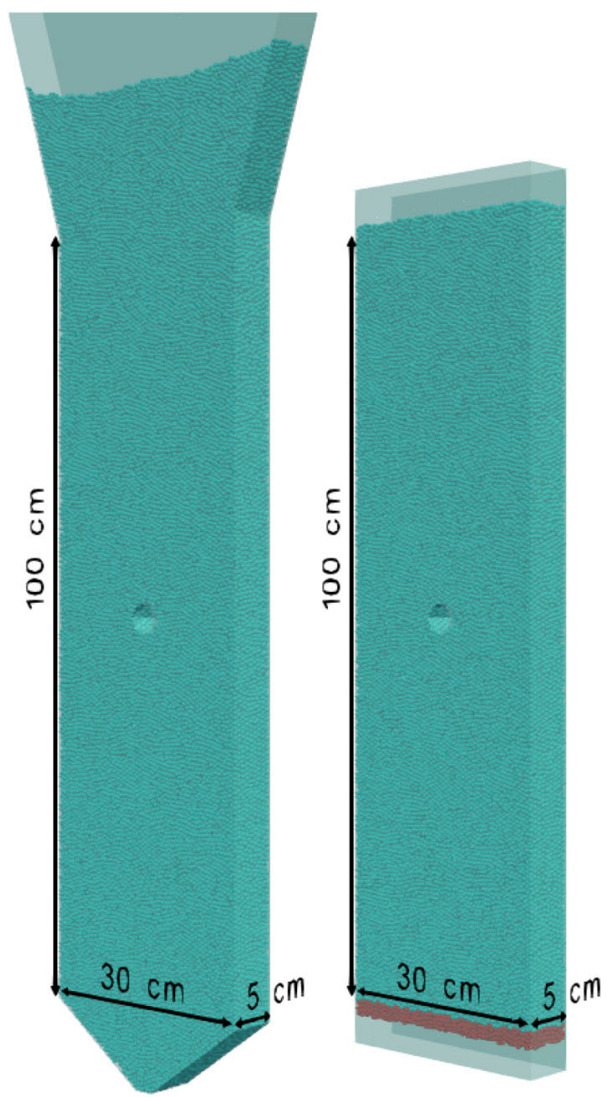

Fig. 2 Left: hopper geometry. Right: channel geometry

geometry allows a better control on the flow velocity, since it can be directly prescribed on the fixed bed. Furthermore, it is more suitable to the simulation of an interstitial fluid because the fluid flow is not disturbed by the change of directions of boundaries.

Although the experiment is quasi-2D, 2D simulations with MigFlow yield values of the drag force much higher than the experimental results. While they capture the velocityindependent behaviour, 3D simulations are necessary for quantitative validation. These 3D simulations with 100000 particles for $40 \mathrm{~s}$ take approximately 40 hours on a SkyLake $2.3 \mathrm{GHz}$ CPU. A sensitivity analysis on the convergence criterion showed that at value of $10^{-6} \mathrm{~m}$ for the geometrical tolerance allows good confidence in the value of the drag force. Figure 3 shows a typical curve for the norm of the drag force versus time. This force is computed as the sum of all the contact impulses on the cylinder over the time step, divided by the time step:

$\mathbf{F}=\frac{1}{\Delta t} \sum_{\beta} m_{\beta}\left(\delta U_{N} \mathbf{n}+\delta \mathbf{U}_{T} \cdot \mathbf{t}\right)$,

where $\beta$ denotes the contacts in which the cylinder is implied and $m_{\beta}$ the mass of the corresponding grains. Remember that 


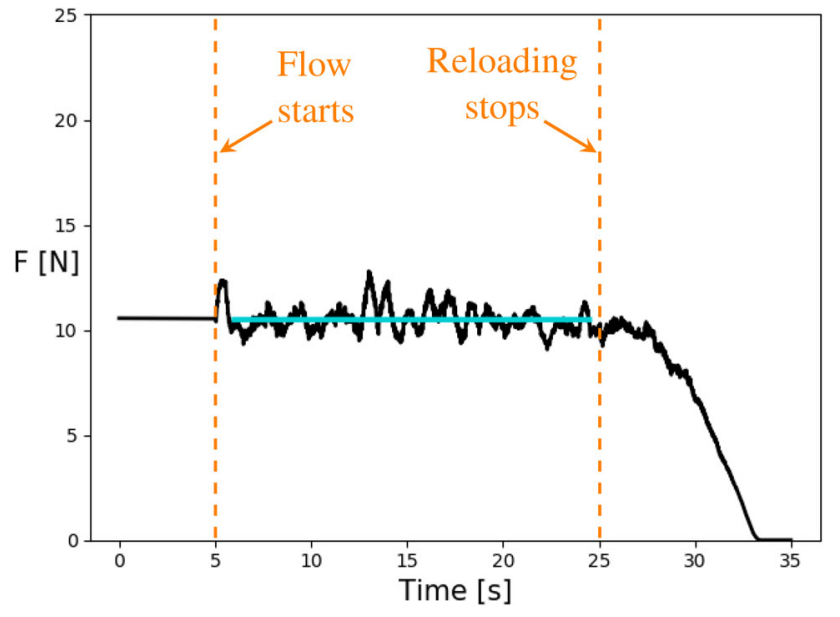

Fig. 3 Typical curve for the drag force, resulting from a moving average with span $t_{a v}=0.5 \mathrm{~s}$ that only takes past data into account. The mean drag force is in blue. The flow velocity is $V=64 \mathrm{~mm} / \mathrm{s}$ and the time step is $.001 \mathrm{~s}$

the velocity corrections are with respect to the free velocities of the grains computed from the external forces. Hence, these are not zero in static situations. The grains are at rest for five seconds before the flow begins, either by opening the aperture in the hopper geometry, or by setting the particle bed in motion in the channel geometry. After a while, the reloading stops and the container empties. The value of the drag force is taken as the average value between the start of the flow and the stop of the reloading. Figure 4 shows a snapshot of the contact network in 2D between the grains when they are flowing. This network fluctuates quickly as contacts are continuously initiated and broken, which is reflected by the variability of the data in Fig. 3.

\subsection{Static stress state}

The value of the static force before the flow begins is difficult to validate as such, because of the complex structure of the contact network. It varies from a simulation to another. Because of this, the focus is put on the static stress inside the granular material, which is linked to the drag force $[16,24]$. The discrete stress tensor can be computed at given grid points directly from the contact forces between the particles:

$\sigma_{i j}=\frac{1}{V} \sum_{\beta \in V} \mathrm{f}_{i}^{\beta} \cdot l_{j}^{\beta}$

where $V$ is a given volume around the grid point, $\mathrm{f}_{i}^{\beta}$ is the $i$ th component of the contact force of contact $\beta$ and $l_{j}^{\beta}$ the $j$ th component of its centre to centre vector. The volume $V$ is chosen as $\pi d^{3} / 6$ and the grid points are separated by a distance $\sqrt{2} d$, with $d=2 r$ the particle diameter.

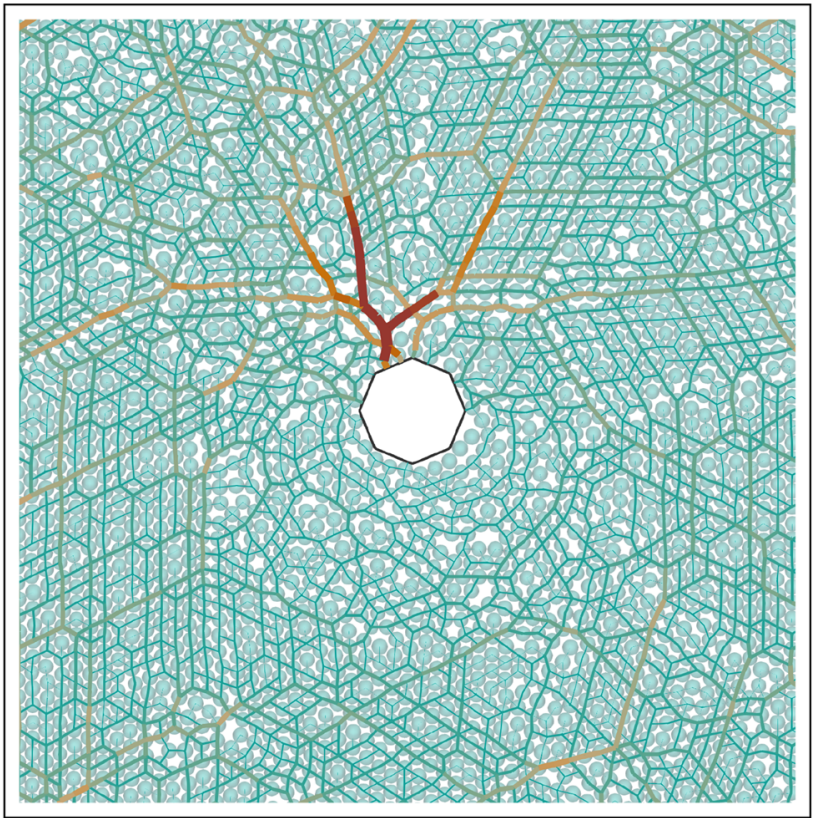

Fig. 4 Snapshot of the contact network in 2D between the grains when they are flowing. The redder and wider the line, the larger the contact force between the two grains

The vertical stress $\sigma_{y y}$ has been shown to asymptote to a saturating value $\sigma_{0}$ as depth increases, unlike hydrostatic stress in newtonian fluids. Indeed, because of the frictional nature of the contacts, arches appear that deflect a part of the weight of the grains onto the side walls. This feature was first modeled by Janssen [29]. He supposed that the horizontal stress was uniform across a vertical section of the container, and that the contact forces of the grains with the walls were at the point of Coulomb failure, i.e. $\mathrm{f}_{T}=\mu_{w} \mathrm{f}_{N}$. He obtained what follows:

$\left\{\begin{array}{l}\sigma_{y y}(y)=\sigma_{0}\left(1-\exp \left(-\frac{H-y}{y_{0}}\right)\right) \\ \sigma_{0}=\phi_{p} \rho_{p} g y_{0}\end{array}\right.$

where $g$ is the norm of the acceleration due to gravity, $H$ the total height of the granular column and $\phi_{p}$ the packing fraction. In all simulations, $\phi_{p}$ was close to the maximum value for spheres in $3 \mathrm{D} \phi_{p} \sim 0.64$. In rectangular containers, the value of $y_{0}$ is given by [16]:

$y_{0}=\frac{1}{K \mu_{w}} \frac{(L-2 r)(W-2 r)}{2(L+W-4 r)}$,

with $L$ and $W$ the length and width of the container, respectively, and $r$ the particle radius. The latter is used to take finite particle size effects into account. For the effect to be significant, the ratios $H / W$ and $H / L$ need to be large enough, which is the case in the considered experiment. The parameter $K$ is called the coefficient of earth pressure. 


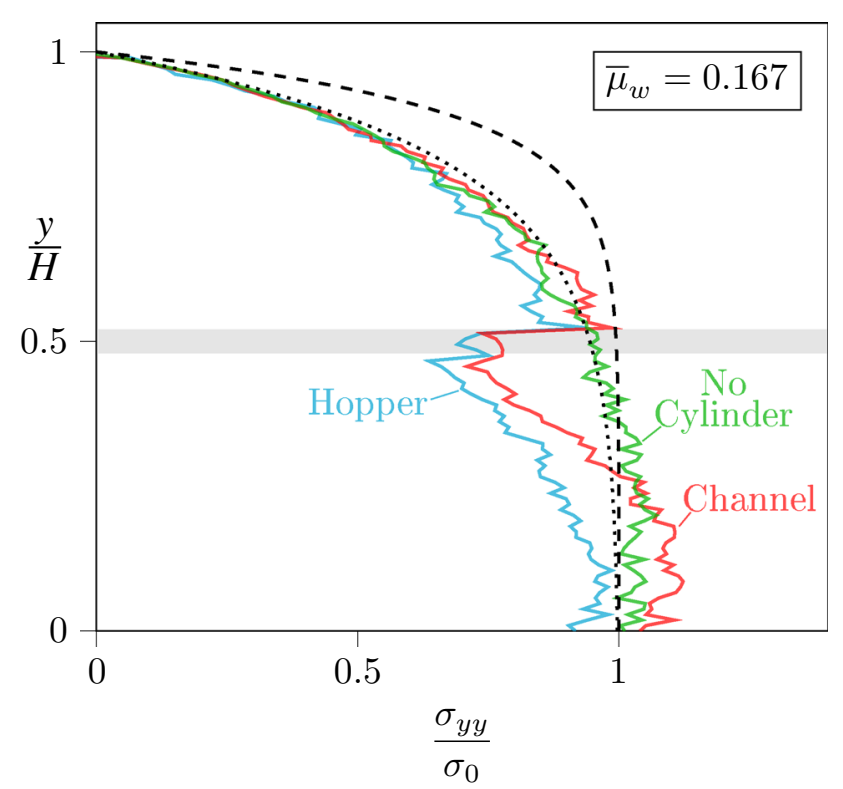

Fig. 5 Width and length averaged vertical stress as a function of height inside the grains before the flow starts. The grey zone indicates the position of the cylinder. Solid curves represent the simulation data. The dashed curve represents Eq. 26 with $y_{0}$ from Eq. 27. The dotted curve is Eq. 26 with $\mu_{w}=\bar{\mu}_{w}$ in Eq. 27

It represents the ratio between the horizontal stress and the vertical stress in a granular material. In a container filled by pouring, it can be assessed as [34]:

$K=\frac{1-\sin (\psi)}{1+\sin (\psi)}$

where $\psi$ is the internal angle of friction of the material. Here, it is estimated $\operatorname{ast}^{-1}\left(\mu_{p}\right)$. If a stress load of magnitude $Q_{0}$ is applied at the top of the granular column, then the Janssen equation must be modified by adding a term $Q(y)$ that takes this load into account [36]:

$Q(y)=Q_{0} \exp \left(-\frac{H-y}{y_{0}}\right)$.

This term is useful to compare stress curves in granular columns with different total heights. The same height bounds are taken for every column so that the relative position of the cylinder is the same, and the stress state above the upper bond is rendered as an applied load $Q_{0}$. The stress profiles obtained with the simulations for the hopper, the channel, and the channel without cylinder are given in Fig. 5.

The curves corresponds to the model from Eq. 26, but with values of $y_{0}$ that are different from Eq. 27. Since $L, W$ and $r$ are purely geometrical, the differences should arise either from $K$ or $\mu_{w}$. The parameter $K$ has a mean value between 0.7 and 0.8 , which is close to the value of 0.74 predicted by Eq. 28. Although the hypothesis of uniform horizontal

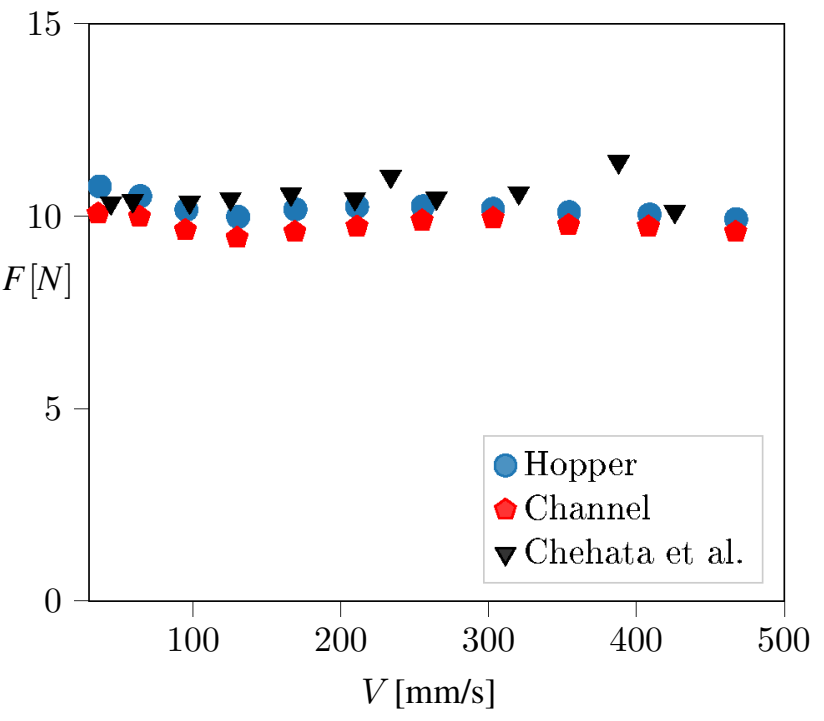

Fig. 6 Comparison of the drag force versus velocity from Chehata et al. with hopper and channel geometry simulations

stress is wrong, the differences between the estimated and the measured value of $K$ are too small to explain the contrast in $y_{0}$. This could actually be due to $\mu_{w}$, because the contacts at the walls are not at the point of Coulomb failure. As the particles could slip on one another, the walls could not support tangential forces much higher than the one between the particles [32]. The fitted effective value of the wall friction coefficient $\bar{\mu}_{w}$ is given in Fig. 5. Such a lower value of effective friction is also observed in the dynamic case, because of the force network fluctuations [10]. As seen in Figs. 5 and 7, the cylinder significantly modifies the stress field inside the grains, and the hopper and channel geometries yield similar results. In Fig. 7, the stress magnitude is lower in the top of the domain for the channel because the total height of grains above the cylinder is lower than with the hopper. However, the difference vanishes as depth increases and the saturating stress is reached before the cylinder. Just before the cylinder, the stress increases and suddenly drops afterwards. This is because the cylinder shields the grains directly below by deflecting the weight of the grains towards the walls. The drop is recovered over three to four cylinder diameters. The cylinder also gives the stress field a semi-circular orientation - indicated by the red lines in Fig. 7 - downstream, which persists for approximately two diameters.

\subsection{Drag force}

When the flow starts, the force exerted on the cylinder changes to reach the regime drag force. Results presented in Fig. 6 are the average over 4 runs, each with new random starting positions for the grains. Both geometries yield results matching the data from Chehata et al., although the channel 


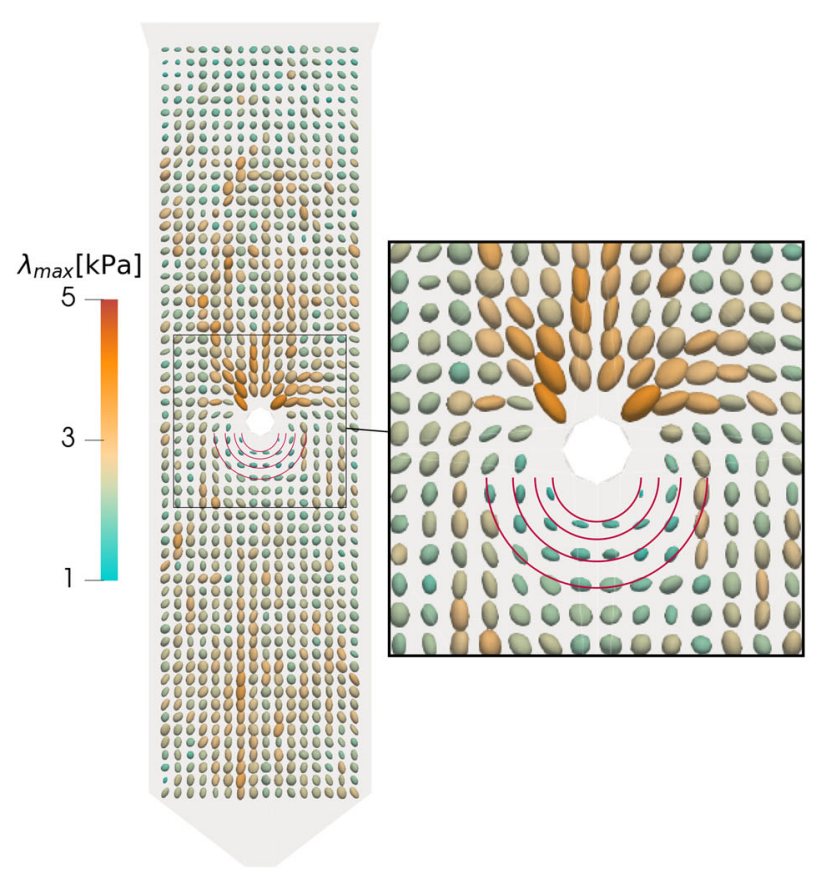

(a)

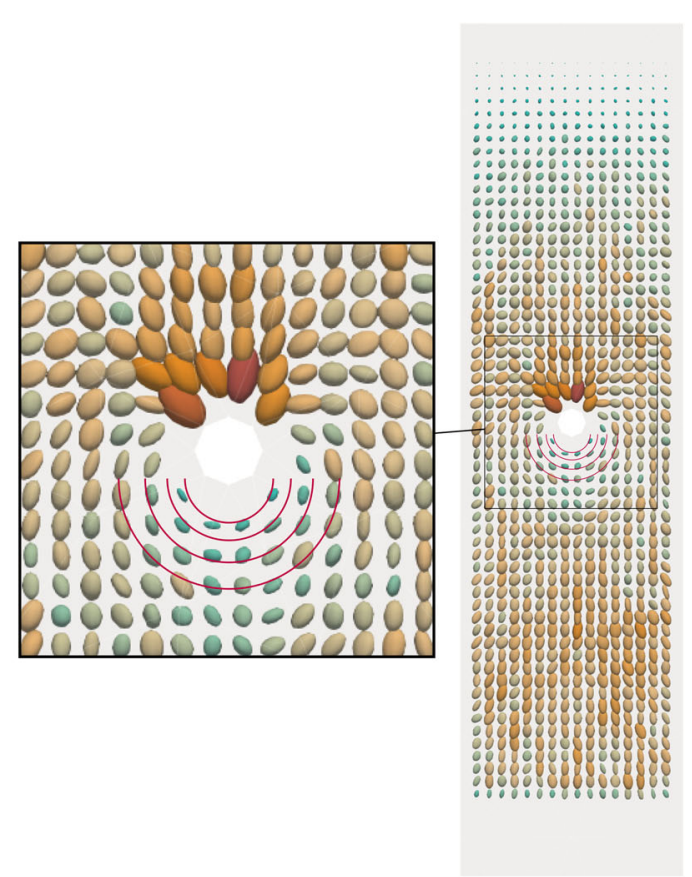

(b)

Fig. 7 Stress field inside the grains before the flow starts. a: hopper geometry. b: channel geometry. The ellipses' axes represent the principal stresses. Red lines underline the semi-circular orientation of the stress field

seems to be slightly below the hopper. The drag force itself has a significant standard deviation but its mean value did not change much over the different runs, with mean standard deviations of $0.031 \mathrm{~N}$ and $0.059 \mathrm{~N}$ for the hopper and the channel respectively. The coefficients of friction were set to $\mu_{p}=0.15$ between the particles and $\mu_{w}=0.3$ between the particles and the walls to obtain matching results. This difference should arise from the hypothesis of the same friction coefficient with the walls and with the cylinder. As detailed in Sect. 4.1, the former tends to reduce the drag force and the latter tends to increase it. Hence, the higher value of $\mu_{w}$ compared to $\mu_{p}$ is a compromise resulting from a competition between those two phenomena. Concerning the stress field, its norm evolves upon flowing of the grains but its orientation remains unchanged. Both norm and orientation do not significantly change with the flowing velocity. The orientation indicates that the drag is mainly due to compressive stress in front of the cylinder, i.e. in the stagnation zone, as supposed by Chehata at al. [16]. The semi-circular shape downstream, see Fig. 7, supports the assumption of a circular zone of influence of the object $[24,46]$.

\section{Results}

The channel geometry yields results similar to the hopper geometry. Because of its simplicity and its lower computational cost, it is used to study the influence of both friction and the interstitial fluid. Friction is at the origin of the velocity- independent behaviour of the drag. It also impacts the drag through the Janssen effect which determines the static stress in contained grains. Many applications of the granular drag - such as industrial mixing or avalanche protecting devices feature an interstitial fluid. Its effects include drag reduction and fluidization at lower velocities.

\subsection{Impact of friction coefficients}

Simulations were performed with a mean velocity of $V=64$ $\mathrm{mm} / \mathrm{s}$ with both friction coefficients ranging independently from 0 to 0.6 by steps of 0.05 . The results are presented in Fig. 8. For a fixed particle friction coefficient $\mu_{p}>0.1$, the drag decreases exponentially as the wall friction coefficient $\mu_{w}$ increases. At low $\mu_{p}<0.1$, the exponential flattens and the drag becomes independent of $\mu_{w}$. For a fixed $\mu_{w}>0.1$, the drag decreases exponentially as $\mu_{p}$ increases. At low $\mu_{w}<$ 0.1 , the drag increases with $\mu_{p}$. Other simulations show that when friction vanishes, inertial effects are predominant and the drag force exhibits a velocity dependent behaviour.

Those behaviours indicate that the drag $F$ is linked to the saturating stress. The latter decreases with increasing friction, as the walls are able to support a larger fraction of the load. This Janssen effect should be taken into account in the choice of the dimensionless quantity $F^{*}$, sometimes referred to as the effective friction $[6,40]$ :

$F^{*}=\frac{F}{P D W}$, 

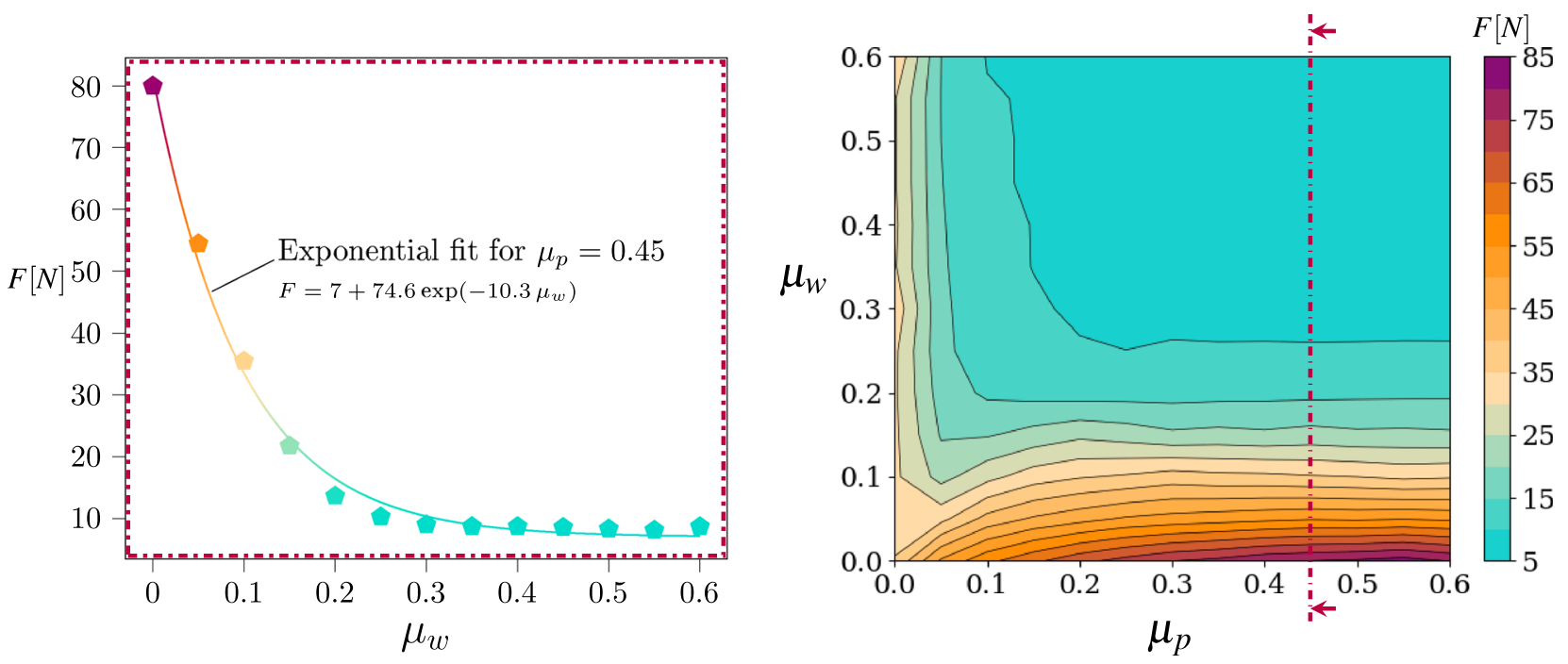

Fig. 8 Left: drag force as a function of $\mu_{w}$ for $\mu_{p}=0.45$. Right: drag force as a function of the friction coefficients

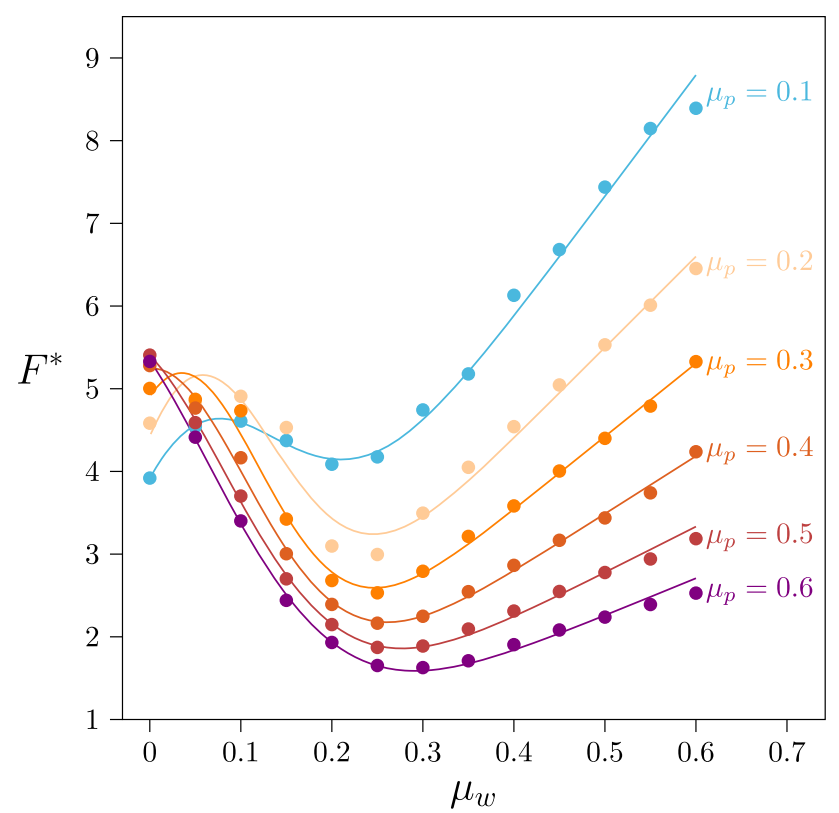

Fig. $9 F^{*}$ as a function of the friction coefficients for different values of $\mu_{p}$. Solid lines are fits from Eq. 33. For clarity, some curves have been omitted

with $P=\sigma_{y y}\left(\mathbf{x}_{c}\right)$ computed with Eq. 26. It is to note that the Janssen effect was shown to remain qualitatively valid in flowing conditions - at least for velocities up to $35 \mathrm{~mm} / \mathrm{s}-$ but with an even stronger screening effect [11]. The exact difference being difficult to compute, the formula for the static case is used. This should not affect the general behaviour of the data, but only its absolute value. $F^{*}$ is plotted as a function of $\mu_{w}$ for different values of $\mu_{p}$ in Fig. 9.

Two regimes can be distinguished. From $\mu_{w}=0.3$ on, $F^{*}$ linearly increases with $\mu_{w}$ with a slope that decreases with increasing $\mu_{p}$. It can be fitted with the following functional form:

$$
F^{*}=\underbrace{A \mu_{w}}_{\text {Cylinder friction }}+B
$$

with A and B fitting parameters functions of $\mu_{p}$. Fitting $A$ for $\mu_{p}$ yields:

$A\left(\mu_{p}\right)=\frac{2.6}{0.074+\mu_{p}}$.

This linear term is associated to the friction between the cylinder and the moving grains. It is directly proportional to $\mu_{w}$, but as $\mu_{p}$ increases, the grain network reorganisation dominates. If the data for $\mu_{p}<0.3$ is taken into account, the constant term in the model becomes a Gauss function:

$$
F^{*}=\underbrace{A \mu_{w}}_{\text {Cylinder friction }}+B \exp \left(-C\left(\mu_{w}-D\right)^{2}\right)
$$

with $C$ and $D$ fitting parameters also functions of $\mu_{p}$.

\subsection{Effect of an interstitial fluid}

The steady flow of a uniform fluid-grain mixture injected under gravity through a channel with a given velocity is simulated. Such circumstances are met in civil engineering applications like raising the level of a building or stabilising its foundations by injecting sand mixed with water [2]. The entrance and the exit of the channel are considered to be very far from the cylinder on which the drag force is measured. No-slip boundary conditions are prescribed on the walls and the cylinder. Results for the drag force are presented in Fig. 10.

Although the force exerted on the cylinder by the fluid is negligible -3 to 4 orders of magnitudes lower than the total drag - the presence of a fluid significantly decreases 

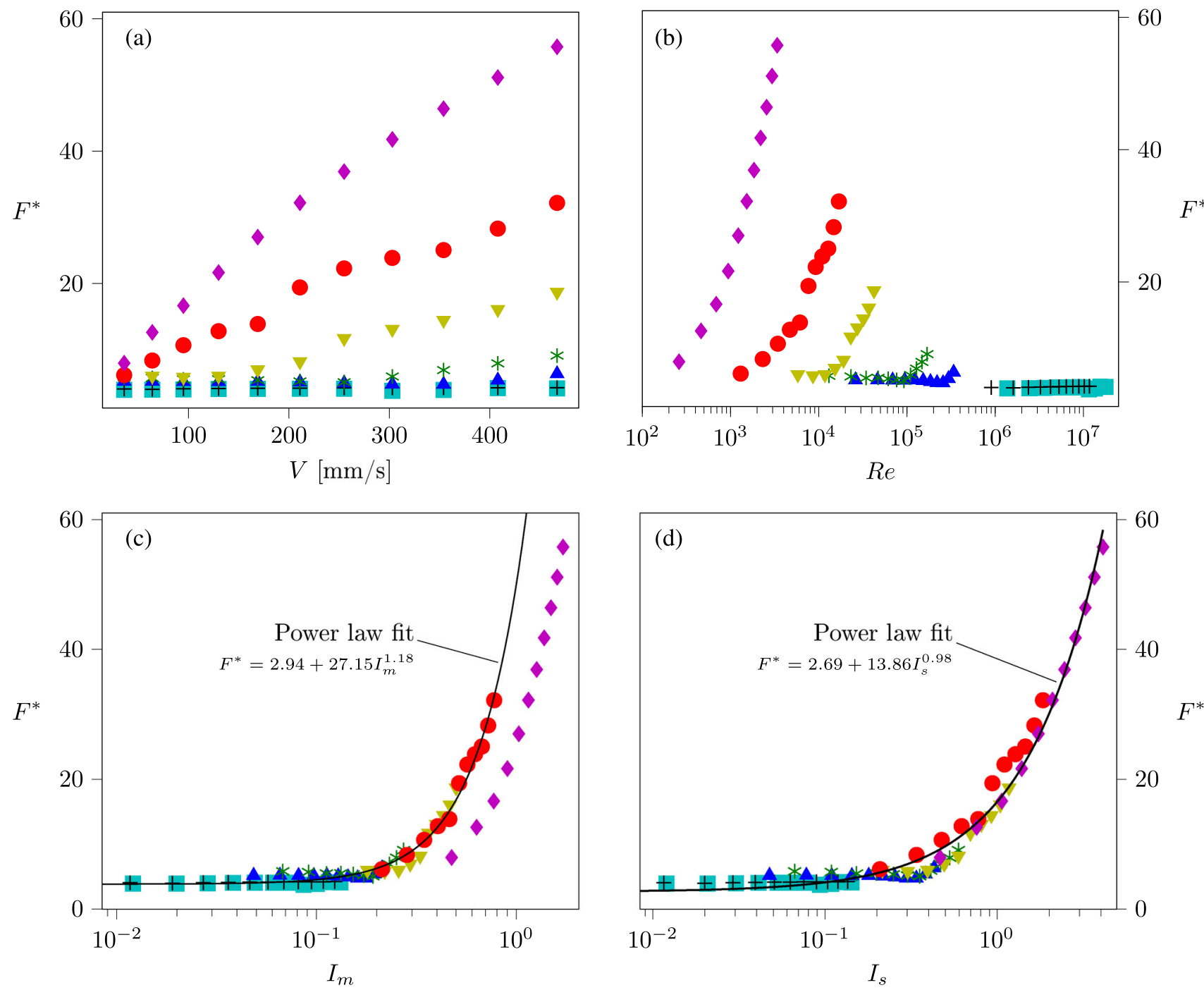

$$
\begin{aligned}
& \square=1000 \eta=.001 \Delta \rho=950 \eta=.05 \nabla \rho=950 \eta=.4 \diamond \rho=950 \eta=5 \\
& +\rho=789 \eta=.0012 * \rho=950 \eta=.1 \quad \rho=950 \eta=1
\end{aligned}
$$

Fig. 10 Dimensionless drag force as a function of different parameters, a: flow velocity, b: Reynolds number, c: modified inertial number, $\mathbf{d}$ : modified viscous number. Densities are in $\mathrm{kg} / \mathrm{m}^{3}$ and viscosities in $\mathrm{Pa} \cdot \mathrm{s}$

the drag force because it modifies the apparent weight of the grains and hence the gravity-induced stress [19]. The same dimensionless quantity $F^{*}$ is computed as in Eq. 30 but corrected for the apparent weight, collapsing the data for different grain densities. The behaviour of the stress tensor indicated a Janssen stress saturation effect that was similar in all simulations. As seen in Fig. 10a, the drag force becomes velocity dependent for a smaller velocity with increasing fluid viscosity. To fully describe the rheology of the fluidgrain mixture, a proper scaling implying both velocity and viscosity is necessary. Figure 10b shows that the Reynolds number $R e=\rho_{f} V D / \eta$ is not sufficient, because it lacks information about the granular part of the mixture. By considering the stresses at stake - inertial $\rho_{p}(d \dot{\gamma})^{2}$, viscous $\eta \dot{\gamma}$, and static $P$ - it is possible to construct a dimensionless number that characterises the flow. The static stress $P$ is computed with Eq. 26 adapted for the apparent weight. It should be noted that the visualisation of the stress tensor showed that the Janssen effect is still valid with a surrounding fluid. Actually, as the saturating stress is smaller because of buoyancy, the static stress and hence Janssen effect should decrease in importance relative to the inertial and viscous stresses.

The so-called modified viscoinertial number is taken as the square root of the ratio of a linear combination of the 


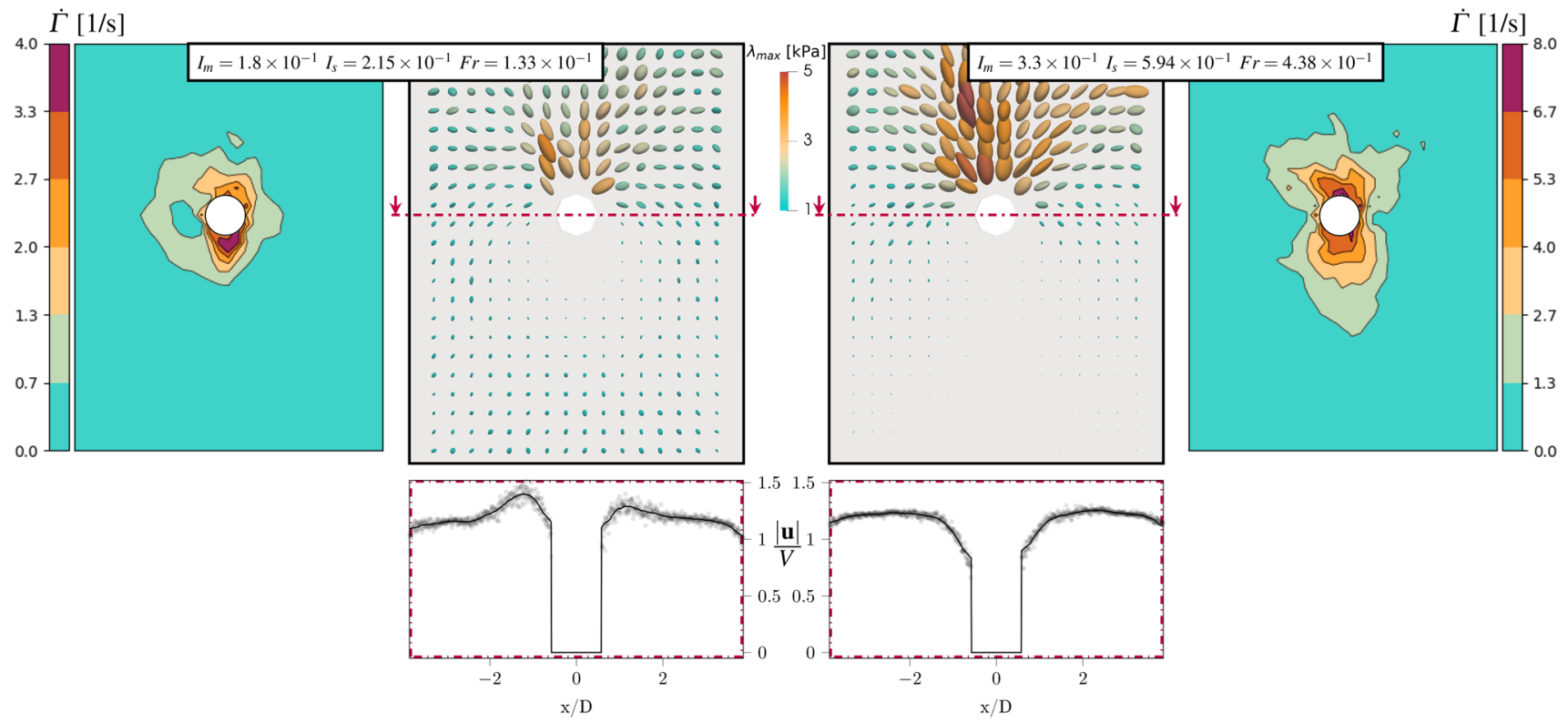

Fig. 11 Grain shear rates $\dot{\Gamma}$, stress fields and normalised velocity $|\mathbf{u}| / V$ profiles near the cylinder. Left: quasi-static regime. Right: fluidized regime. The average shear rate is computed with the same grid than the stress tensor. The grey dots in the velocity profiles are the average

inertial and the viscous stress to the static stress [7,47]:

$I_{m}=\sqrt{\frac{\rho_{p}(d \dot{\gamma})^{2}+\beta \cdot \eta \dot{\gamma}}{P}}$,

where $\dot{\gamma}$ is the shear rate and $\beta$ is a fitting parameter that should depend on material properties such as particle shape and size distribution and friction coefficients [47]. In granular flows, the shear rate may depend on both the cylinder diameter and the grain diameter when the ratio of the two is small [42]. However, since the ratio $D / d$ is around 6.35, the grain diameter is neglected in the shear rate which is chosen as $\dot{\gamma}=V / D$. Nonetheless, the grain diameter is already taken into account in $I_{m}$. The value of $\beta$ that best fits the data is 39.9. As seen in Fig. 10c, this allows a collapse of all curves except the one with the highest viscosity. Ignoring the latter, the data is fitted by a single power law given in the figure. It is possible to collapse all the data by considering shear thickening, which has been observed for immersed granular flows [23]. In this case, the viscosity depends on the shear rate as follows:

$\eta_{s}=H \dot{\gamma}^{n}$,

with $H$ a constant having appropriate units. The value of $n$ that best collapses the data is $n=0.7$. Replacing $\eta$ by $\eta_{s}$ to velocity at grid points. The grid stretches across the whole width of the channel horizontally, and one diameter above and below the cylinder vertically. The black line is the corresponding smoothed curve

obtain a shear-thickening viscoinertial number:

$I_{S}=\sqrt{\frac{\rho_{p}(d \dot{\gamma})^{2}+\beta \cdot \eta_{s} \dot{\gamma}}{P}}$,

yields Fig. 10d. Again, the data can be fitted with a single power law. Several physical mechanisms could explain the shear thickening of dense granular suspensions. Heussinger suggested that it is due to energy dissipation, notably through the formation of hydrodynamic clusters increasing viscous dissipation [26]. These clusters have a size linked to the viscosity of the surrounding fluid, which could explain why the data with the highest viscosity is the most affected by the consideration of shear thickening. Others suggested that it originates from constrains on particle rotation and relative sliding. These constrains can come from interparticle friction [43] but also from hydrodynamic forces [28]. As hydrodynamic forces increase with viscosity, this could again explain the higher sensitivity of the high viscosity data set to shear thickening. However, it is still unclear how shear thickening manifests in the simulations and if $I_{S}$ should be used, or if the highest viscosity data set is at the limit of the numerical model and $I_{m}$ should be used.

The transition between the quasi-static $-F^{*}=$ constant - and the fluidized regimes occurs between $I_{m}=0.15$ and $I_{m}=0.4$ or between $I_{s}=0.3$ and $I_{s}=0.6$. The corresponding Froude numbers corrected for the apparent gravity due to buoyancy $-F r=V / \sqrt{\left(1-\rho_{f} / \rho_{p}\right) g D}$ - are below 
the threshold value of 10 . This confirms that the fluid causes fluidization at lower velocities. Figure 11 shows the grain shear rate, the stress state and the velocity profiles in the quasi-static and the fluidized regimes close to the transition.

The grain shear rate is computed as the Frobenius norm of the deformation rate tensor :

$$
\dot{\Gamma}=\sqrt{\sum_{i} \sum_{j}\left(\frac{1}{2} \frac{\partial u_{i}}{\partial x_{j}}+\frac{1}{2} \frac{\partial u_{j}}{\partial x_{i}}\right)^{2}} .
$$

The velocities are evaluated on a grid as the average of the velocities of the grains near the grid points. Their derivatives are computed with a first order central finite difference scheme. The grid is the same than the one used to compute the stress tensor. The shear zone size increases as expected with the flow velocity, but its shape changes from roughly circular in the quasi-static regime to an elongated shape aligned with the flow direction in the fluidized regime. Fluidization decreases the stress field downstream of the cylinder, implying a reduction of the amount of interparticle contacts. In the quasi static regime, the increase of velocity occurs in the vicinity of the cylinder only: the flow slips in this region [16]. In the fluidized regime, the velocity increase is uniform across the channel and decays near the cylinder, which is characteristic of a viscous flow.

\section{Conclusion}

The granular flow around a cylinder was simulated in both dry and immersed cases and the drag force was measured. The granular phase was solved with the nonsmooth contact dynamics method: the particles are tracked in a Lagrangian way and interpenetrations are prevented by correcting their velocities following a perfectly inelastic collision law, taking friction into account. The fluid phase was solved with a Finite Element Method based on an averaging process of the fluid fields according to the fluid volume fraction. Both solvers are coupled through a predictor-corrector scheme.

The model and a channel simulation geometry were validated in the dry case against physical experiments. The Janssen effect was captured with an effective wall friction coefficient closer to the particle friction coefficient. The independent behaviour of the drag force versus velocity was quantitatively recovered. Visualization of the stress field showed that the drag force is mainly due to compressive stress upstream of the cylinder. Its circular orientation downstream of the cylinder supports the hypothesis of a spherical zone of influence of the penetrating object.

The impact of the wall and particle friction coefficients was studied. The drag force was found to decrease exponentially with the friction coefficients, as the walls take a larger part of the gravitational load. A scaling for the drag force was then proposed based on the Janssen effect. It was found that the dimensionless drag force as a function of the wall friction coefficient follows two regimes. For high wall friction, the drag increases linearly with the wall friction coefficient, with a slope decreasing with increasing particle friction. For low wall friction, the drag follows a Gaussian function with parameters depending on particle friction. A more accurate study of the physical phenomenon of this contribution is required to fully characterise it.

In the immersed case, the contribution of the fluid on its own was found negligible compared to the one of the grains. Two dimensionless numbers were used to characterise the flow. The modified viscoinertial number $I_{m}$, based on a ratio of relevant stresses yielded a good collapse of the data except for the highest fluid viscosity. Considering shear thickening and using an adapted viscoinertial number $I_{s}$ yielded a collapse of the whole data. Further investigation should be led to determine if $I_{m}$ or $I_{s}$ should be preferred. The transition between the quasi-static and the fluidized regime was accompanied by a reduction of the interparticle contacts downstream of the cylinder. The shear zone changes from circular to elongated in the direction of the flow. The velocity profile shifted from a slipping behaviour in the vicinity of the cylinder to a viscous one with a uniform velocity increase across the channel section and a decay close to the cylinder.

Acknowledgements Computational resources have been provided by the supercomputing facilities of the Université catholique de Louvain (CISM/UCL) and the Consortium des Équipements de Calcul Intensif en Fédération Wallonie Bruxelles (CÉCI) funded by the Fond de la Recherche Scientifique de Belgique (F.R.S.-FNRS) under convention 2.5020.11 and by the Walloon Region

Code Availability The source code of the MigFlow software used to produce the results presented in this article is released under the GPL license and available here https://git.immc.ucl.ac.be/fluidparticles/migflow/-/ tags/GranularDrag2

\section{Declarations}

Conflict of interest The authors declare that they have no conflict of interest.

\section{References}

1. Acary V (2013) Projected event-capturing time-stepping schemes for nonsmooth mechanical systems with unilateral contact and coulomb's friction. Comput Methods Appl Mech Engrg 256:224250

2. Affeld K, Affeld F, Debaene P, Goubergrits L (2006) Injection of granular material. J Visualization 9:31-38

3. Alam M, Luding S (2002) Rheology of bidisperse granular mixture via event driven simulations. J Fluid Mech 476:69-103 
4. Alart P (2014) How to overcome indetermination and interpenetration in granular systems via nonsmooth contact dynamics. an exploratory investigation. Comput Methods Appl Mech Engrg 270:37-56

5. Albert R, Pfeifer M, Barabasi A, Schiffer P (1999) Slow drag in a granular medium. Phys Rev Lett 82:205-208

6. Allen B, Kudrolli A (2019) Effective drag of a rod in fluid-saturated granular beds. Phys Rev E 100:022901

7. Amarsid L, Delenne J, Mutabaruka P, Monerie Y, Perales F, Radjai F (2017) Viscoinertial regime of immersed granular flows. Phys Rev E 96:012901

8. Anderson TB, Jackson R (1967) A fluid mechanical description of fluidized beds. Ind Eng Chem Fundam 6:527-539

9. Artoni R, Loro G, Richard P, Gabrieli F, Santomaso A (2019) Drag in wet granular materials. Powder Technol 356:231-239

10. Artoni R, Richard P (2015) Effective wall friction in wall-bounded 3d dense granular flows. Phys Rev Lett 115:158001

11. Bertho Y, Giorgiutti-Dauphiné F, Hulin JP (2003) Dynamical janssen effect on granular packing with moving walls. Phys Rev Lett 90:144301

12. Bizon C, Shattuck M, Swift J, Swinney H (1999) Transport coefficients for granular media from molecular dynamics simulations. Phys Rev E 60:4340-51

13. Boyer F, Guazzelli E, Pouliquen O (2011) Unifying suspension and granular rheology. Phys Rev Lett 107:188301

14. Campbell C (1990) Rapid granular flows. Annu Rev Fluid Mech 22:57-92

15. Capecelatro J, Desjardins O (2013) An Euler-Lagrange strategy for simulating particle-laden flows. J Comput Phys 238:1-31

16. Chehata D, Zenit R, Wassgren C (2003) Dense granular flow around an immersed cylinder. Phys Fluids 15:1622-1631

17. Constant M, Coppin N, Dubois F, Vidal V, Legat V, Lambrechts J (2020) Simulation of air invasion in immersed granular beds with an unresolved FEM-DEM model. Comp Part Mech. https://doi. org/10.1007/s40571-020-00351-4

18. Constant M, Dubois F, Lambrechts J, Legat V (2018) Implementation of an unresolved stabilised FEM-DEM model to solve immersed granular flows. Comp Part Mech 5:1-14. https://doi.org/ 10.1007/s40571-018-0209-4

19. Costantino DJ, Bartell J, Scheidler K, Schiffer P (2011) Low velocity granular drag in reduced gravity. Phys Rev E 83:011305

20. Cundall P, Strack O (1979) A discrete numerical model for granular assemblies. Géotechnique 29:47-65

21. Dallavalle JM, Klemin A (1943) Micromeritics?: the technology of particles. Pitman Publishing Corporation, London

22. Deen NG, Van Sint Annaland M, Van der Hoef MA, Kuipers JAM (2007) Review of discrete particle modeling of fluidized beds. Chem Eng Sci 62:28-44

23. Fall A, LemaItre A, Bertrand F, Bonn D, Ovarlez G (2010) Shear thickening and migration in granular suspensions. Phys Rev Lett 105:268303

24. Faug T (2015) Macroscopic force experienced by extended objects in granular flows over a very broad Froude number range. Eur Phys J E 38:34

25. Gidaspow D (1994) Multiphase flow and fluidization?: continuum and kinetic theory description. Academic Press, Cambridge

26. Heussinger C (2013) Shear thickening in granular suspensions: interparticle friction and dynamically correlated clusters. Phys Rev E 88:050201

27. Hu H, Joseph D, Crochet M (1992) Direct simulation of fluid particle motion. Theor Comput Fluid Dyn 3:285-306

28. Jamali S, Brady JF (2019) Alternative frictional model for discontinuous shear thickening of dense suspensions: Hydrodynamics. Phys Rev Lett 123:138002

29. Janssen HA (1895) Versuche uber getreidedruck in silozellen. Zeitschr. d. Vereines deutscher Ingenieure 39:1045
30. Jean M (1999) The non-smooth contact dynamics method. Computer Methods Appl Mech Eng 177:235-257

31. Jean M (2010) Méthode de dynamique des contacts. In: Dubois $\mathrm{F}$, Radjai $\mathrm{F}$ (eds) Modélisation numérique discrète des matériaux granulaires. Hermès-Lavoisier, Paris, pp 51-82

32. Landry JW, Grest GS, Silbert LE, Plimpton SJ (2003) Confined granular packings: structure, stress and forces. Phys Rev E 67:041303

33. Lun C, Savage S, Jeffrey D, Chepurniy N (1984) Kinetic theories for granular flows?: inelastic particles in couette flow and slightly inelastic particles in a general flowflied. J Fluid Mech 140:223-256

34. Mesri G, Hayat TM (1993) The coefficient of earth pressure at rest. Can Geotech J 30:647-666

35. Moreau J (2004) An introduction to unilateral dynamics. In: Frémond M, Maceri F (eds) Novel approaches in civil engineering. Springer, Berlin, pp 1-46

36. Nedderman RM (1992) Statics and kinematics of granular materials. Cambridge University Press, Cambridge

37. Panaitescu A, Clotet X, Kudrolli A (2017) Drag law for an intruder in granular sediments. Phys Rev E 95:032901

38. Peskin C (2002) The immersed boundary method. Acta Numer 11:479-517

39. Pouliquen O, Cassar C, Jop P, Forterre Y, Nicolas M (2006) Flow of dense granular material: towards simple constitutive laws. J Stat Mech: Theory Exp 2006(07):P07020-P07020. https://doi.org/10. 1088/1742-5468/2006/07/p07020

40. Pouliquen O, Forterre Y (2009) A non-local rheology for dense granular flows. Philos Trans R Soc A 367:5091-5107

41. Richardson J, Zaki W (1954) The sedimentation of a suspension of uniform spheres under conditions of viscous flow. Chem Eng Sci 3:65-73

42. Seguin A, Bertho Y, Martinez F, Crassous J, Gondret P (2013) Experimental velocity fields and forces for a cylinder penetrating into a granular medium. Phys Rev E 87:012201

43. Seto R, Mari R, Morris JF, Denn MM (2013) Discontinuous shear thickening of frictional hard-sphere suspensions. Phys Rev Lett 111:218301

44. Sovilla B, Kern M, Schaer M (2010) Slow drag in wet-snow avalanche flow. J Glaciol 56:587-592

45. Takehara Y, Okumura K (2014) High-velocity drag friction in granular media near the jamming point. Phys Rev Lett 112:148001

46. Tordesillas A, Hilton JE, Tobin ST (2014) Stick-slip and force chain evolution in a granular bed in response to a grain intruder. Phys Rev E 89:042207

47. Trung Vo T, Nezamabadi S, Mutabaruka P, Delenne J, Radjai F (2020) Additive rheology of complex granular flows. Nat Commun $11: 1476$

48. Tsuji Y, Kawaguchi T, Tanaka T (1993) Discrete particle simulation of two-dimensional fluidized bed. Powder Technol 77:79-87

49. Wassgren C, Karion A, Cordova J, Zenit R (2003) Dilute granular flow around an immersed cylinder. Phys Fluids 15:3318-3330

50. Wen CY, Yu HY (1966) Mechanics of fluidization. Chem Eng Prog Symp Ser 62:100-111

51. Wieghardt K (1975) Experiments in granular flow. Annu Rev Fluid Mech 7:89-114

52. Zhang J, Fan L, Zu C, Pfeffer R, Qi D (1999) Dynamic behavior of collision of elastic spheres in viscous fluids. Powder Technol 106:98-109

53. Zhou F, Advani SG, Wetzel ED (2004) Slow drag in granular materials under high pressure. Phys Rev E 69:061306 